\title{
Supporting Information: Nonlinear transient and steady state stretching of deflated vesicles in flow
}

\author{
Dinesh Kumar ${ }^{1,2}$ and Charles M. Schroeder ${ }^{1,2,3, \text { 冈 }}$ \\ ${ }^{1}$ Department of Chemical and Biomolecular Engineering \\ University of Illinois at Urbana-Champaign, Urbana, IL, 61801 \\ ${ }^{2}$ Beckman Institute for Advanced Science and Technology \\ University of Illinois at Urbana-Champaign, Urbana, IL, 61801 \\ ${ }^{3}$ Department of Materials Science and Engineering \\ University of Illinois at Urbana-Champaign, Urbana, IL, 61801
}

(Dated: September 4, 2021)

\section{Supplementary TEXT}

\section{Critical capillary number for pulling out all the membrane fluctuations}

For the stretching experiments in our work, vesicles are deformed in the bending regime where microscopic membrane fluctuations are pulled out by the external flow. Thus, the volume and area of vesicle remains conserved during the time scale of experiments. Here, we estimate the critical capillary number required to pull out all membrane undulations in terms of vesicle material properties. Prior works have described the cross-over membrane tension at the transition from bending to the area-dilation regime [1, 2. Clearly, the vesicle deformation will transition to the area dilation regime once all the membrane folds are pulled out. The membrane tension corresponding to pulling out all the membrane fluctuations is written as $\sigma_{c}=K_{a} k_{B} T / 8 \pi \kappa$, where $K_{a}$ is the area stretching modulus, $k_{B}$ is the Boltzmann constant, $T$ is the temperature [3]. Using force balance, the critical strain rate required to pull out all the membrane folds in our experiments can be expressed in terms of the cross-over tension as:

$$
\dot{\epsilon}_{c}=\sqrt{\frac{2 \sigma_{c} \kappa}{9 \mu^{2} R_{v_{1}}^{4}}}
$$

Thus, the predicted critical capillary number for pulling all the membrane folds is $C a_{c_{3}}=\frac{\mu \dot{\epsilon}_{c} R^{3}}{\kappa}$ which can be expressed as:

$$
C a_{c_{3}}=\frac{R^{3}}{3 \kappa} \sqrt{\frac{2 \sigma_{c} \kappa}{R_{v_{1}}^{4}}}
$$

where $R$ is the equivalent radius of vesicle. Clearly, the critical capillary number depends on vesicle size and material properties namely the bending modulus and area-stretching modulus. For nearly spherical DOPC vesicles $(\nu>0.9)$, the values of $\kappa$ and $K_{a}$ are available in literature. For the quasi-spherical vesicle in Fig. S2, we use the experimental parameters $R \sim R_{v_{1}}=9.9 \mu m, K_{a}=0.1 \mathrm{~N} / \mathrm{m}$, and $\kappa=22.3 k_{B} T$, which provides an estimate for $\dot{\epsilon}_{c} \approx 17.5$ and $C a_{c_{3}} \approx 205$. This predicted value of $C a_{c_{3}}$ from Eq. (S1) is reasonably close to the experimentally observed capillary number $(C a \approx 200)$ at which the vesicle aspect ratio $A_{r}$ saturates (Fig. S2). As shown in Fig. S2, the vesicle aspect ratio $A_{r}$ initially increases linearly with $C a$ upto $C a \approx 50$, and then gradually plateaus at $C a \approx 200$. Thus, the initial linear regime for quasi-spherical steady stretch ends at $\approx 25 \%$ of the capillary number at which the vesicle stretch saturates once all the fluctuations are pulled out. Based on these observations for quasi-spherical vesicles, we aim to estimate the critical capillary number for pulling out all the membrane fluctuations for anisotropic vesicles having $\nu<0.75$.

The analysis for anisotropic vesicles is challenging because the data for bending modulus $\kappa$ and area-stretching modulus $K_{a}$ for anisotropic vesicles is not available. While we characterized the values of $\kappa$ as a function of reduced volume in the main text, the data for $K_{a}$ is still not available. In the absence of this information, we assume that the values of $K_{a}$ for anisotropic vesicles will decrease by the same factor as the bending modulus, when compared to 


\begin{tabular}{|c|c|c|c|}
\hline$\nu$ & $\begin{array}{c}C a_{c_{2}} \\
(\text { obs. })\end{array}$ & $\begin{array}{c}C a_{c_{3}} \\
(\mathrm{pred})\end{array}$ & $\begin{array}{c}\left(\mathrm{Ca}_{c_{2}} / \mathrm{Ca}_{c_{3}}\right) \times 100 \\
(\%)\end{array}$ \\
\hline 0.37 & 5.5 & 15.8 & 34.7 \\
\hline 0.46 & 8.0 & 21.8 & 37.0 \\
\hline 0.54 & 11.2 & 34.1 & 32.8 \\
\hline 0.59 & 14.0 & 31.4 & 44.6 \\
\hline 0.67 & 19.7 & 23.9 & 82.3 \\
\hline 0.73 & 73.9 & 165.9 & 44.5 \\
\hline 0.74 & 69.0 & 103.6 & 66.5 \\
\hline 0.91 & $\mathrm{NA}$ & 206 & $\mathrm{NA}$ \\
\hline
\end{tabular}

TABLE S1. Comparison between experimentally observed $C a_{c_{2}}$ for $A_{r}$ divergence and predicted capillary number $C a_{c_{3}}$ for $A_{r}$ saturation.

their quasi-spherical analogues.

For all the vesicles with reduced volume $\nu<0.75$, we never observed the saturation of steady-state stretch $A_{r}$, due to finite field-of-view of the microscope. Nevertheless, we observed that $A_{r}$ diverges in flow at a critical capillary numbers $\mathrm{Ca}_{c_{2}}$, which is atleast $25 \%$ of the predicted capillary number for unfolding all the thermal fluctuations at which $A_{r}$ presumably saturates in flow. Table S1).

\section{Maximum nanotube length of vesicles under flow}

The maximum length of nanotube when all the membrane fluctuations have been pulled out can be estimated by using the reduced volume definition, and expressing the volume and area of dumbbell shapes in terms of vesicle geometrical parameters. The reduced volume is written as $\nu=3 V \sqrt{4 \pi} / A^{3 / 2}$. Expressing the area of dumbbell shapes as $A=4 \pi R_{v_{1}}^{2}+4 \pi R_{v_{2}}^{2}+2 \pi r_{t} L_{t}$ and volume as $V=4 / 3 \pi R_{v_{1}}^{3}+4 / 3 \pi R_{v_{2}}^{3}+\pi r_{t}^{2} L_{t}$, we can deduce the following equation for estimating the maximum nanotube length:

$$
4 \nu\left(R_{v_{1}}^{2}+R_{v_{2}}^{2}+\frac{r_{t} L_{t}}{2}\right)=4 R_{v_{1}}^{3}+4 R_{v_{2}}^{3}+3 r_{t}^{2} L_{t}
$$

In our experiments, the vesicle nanotube length will keep increasing with an increase in capillary number and the nanotube radius will decrease until it becomes thin enough that the vesicle will rupture. Since the membrane thickness is $\approx 5 \mathrm{~nm}$, we believe that the vesicle will rupture when the nanotube radius becomes $\approx 5 \mathrm{~nm}$. So, we assume that the maximum nanotube length will be achieved when tube radii becomes $10 \mathrm{~nm}$. After this, the vesicle will transition to the area-stretching regime, and will eventually rupture. By fixing $r_{t}=10 \mathrm{~nm}$ in Eq. (S2), we can estimate the maximum nanotube length at the end of bending regime for vesicles in our experiments. For all the vesicles in our experiments, we estimate that the maximum nanotube lengths lie between $\approx 3-10 \mathrm{~mm}$. Thus, the anisotropic vesicles are highly extensible and can stretch upto 100-120 times their equilibrium size in flow.

\section{Model for vesicle steady state stretch}

In order to derive the general model for a wide range of reduced volumes, we assumed a simplified vesicle geometry at the onset of conformational change, irrespective of the initial equilibrium shape (Fig. S8). We note that this is a reasonable approximation for vesicles with reduced volume $\nu>0.5$ as seen in the experimental movies (Movie S1). Thus, the initial base state for all vesicles with $\nu>0.5$ is two equal/unequal spherical bulbs whose observable radii remains constant during the entire stretching event. Since the bending modulus remains same for a vesicle throughout the stretching phase, we can assume any conformation during the process as a base state without losing generality. For vesicles with $\nu<0.5$ in this paper, the assumption of simplified geometry in Fig. S8 no longer holds. Generally, vesicles with $\nu<0.5$ involve a short-length tube connecting two spherical bulbs. So, the area contribution from short-length tube should be considered for calculating the area $A_{0}$ of the base state. 
The model in Equation 3 of the main text is simple, and does not includes the effect of subtle changes in membrane mechanics of highly deflated vesicles. First, the model ignores thermal fluctuations on the membrane nanotubes connecting two spherical ends. Since lower reduced volume vesicles display enhanced thermal fluctuations [4 6] SI Movie 3), this effect may become important for $\nu<0.7$. Secondly, the model does not feature contribution from membrane spontaneous curvature. Prior studies [1, 6, 7] have shown that such effect may be important for highly anisotropic vesicles considered in our experiments. Thirdly, the contribution from area-difference elasticity [8, 9] may be important for large tube lengths $(>400 \mu \mathrm{m})$ considered in our experiments. Thus, we conclude that vesicles with

$\nu<0.7$ undergo extreme nonlinear deformation within a short range of strain rate that is not captured by model accurately.

\section{Supplementary Movies}

Movie 1 - Transient stretching dynamics of a single vesicle in extensional flow: Movie showing the shape evolution of a moderately deflated vesicle with $\nu=0.64$ at $C a=23$ forming elongated asymmetric dumbbell under flow. After the flow is stopped, the vesicle completely relaxes back to its equilibrium shape.

Movie 2 - Enhanced thermal fluctuations of a tubular vesicle: Movie showing thermal fluctuations of a tubular vesicle with $\nu=0.3$ imaged with $63 \mathrm{x}$ oil immersion objective. 


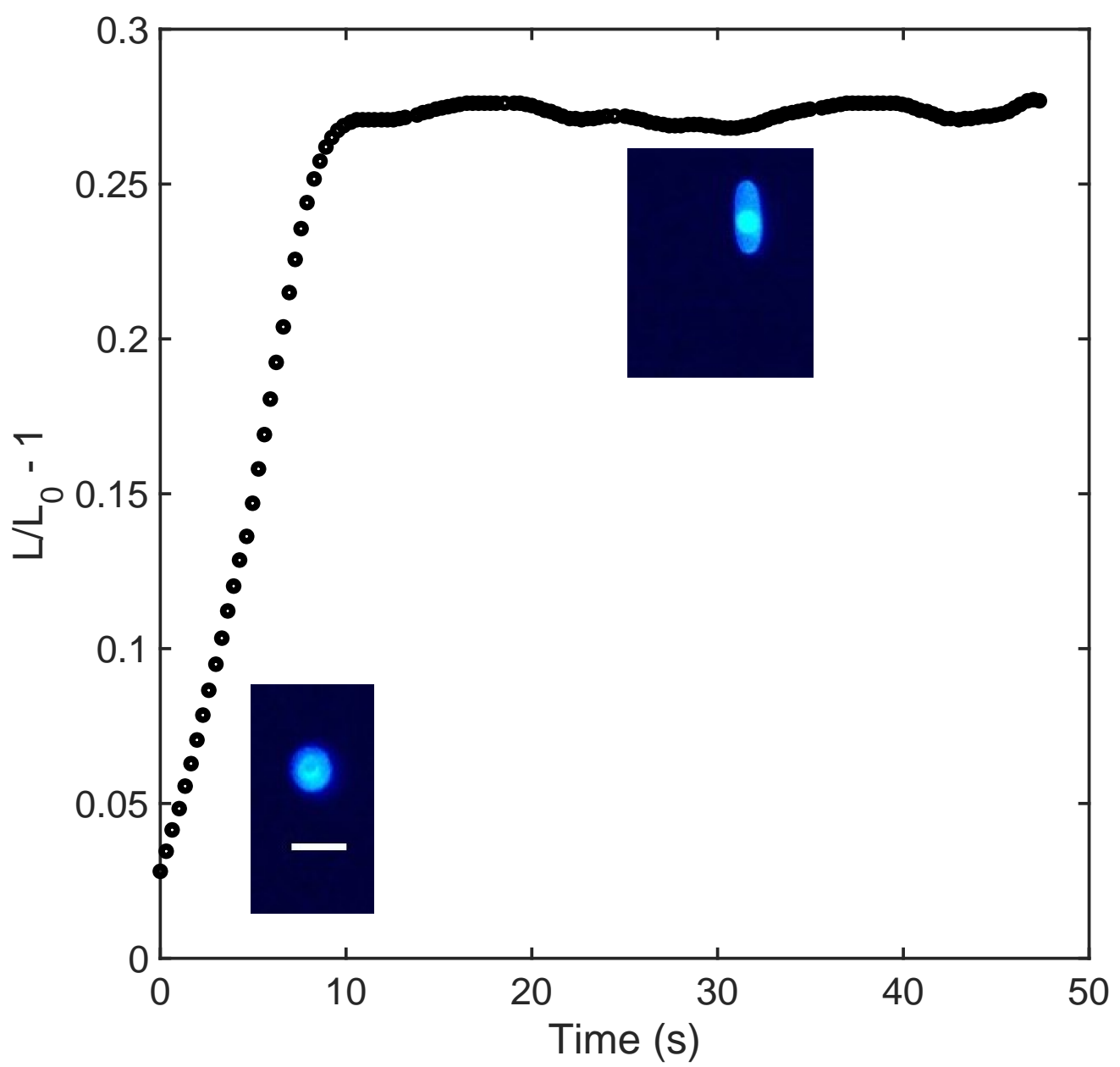

FIG. S1. Transient stretching deformation of a single GUV having reduced volume $\nu=0.95$ under a step-strain with Ca=80. Scale bar is $10 \mu \mathrm{m}$. 


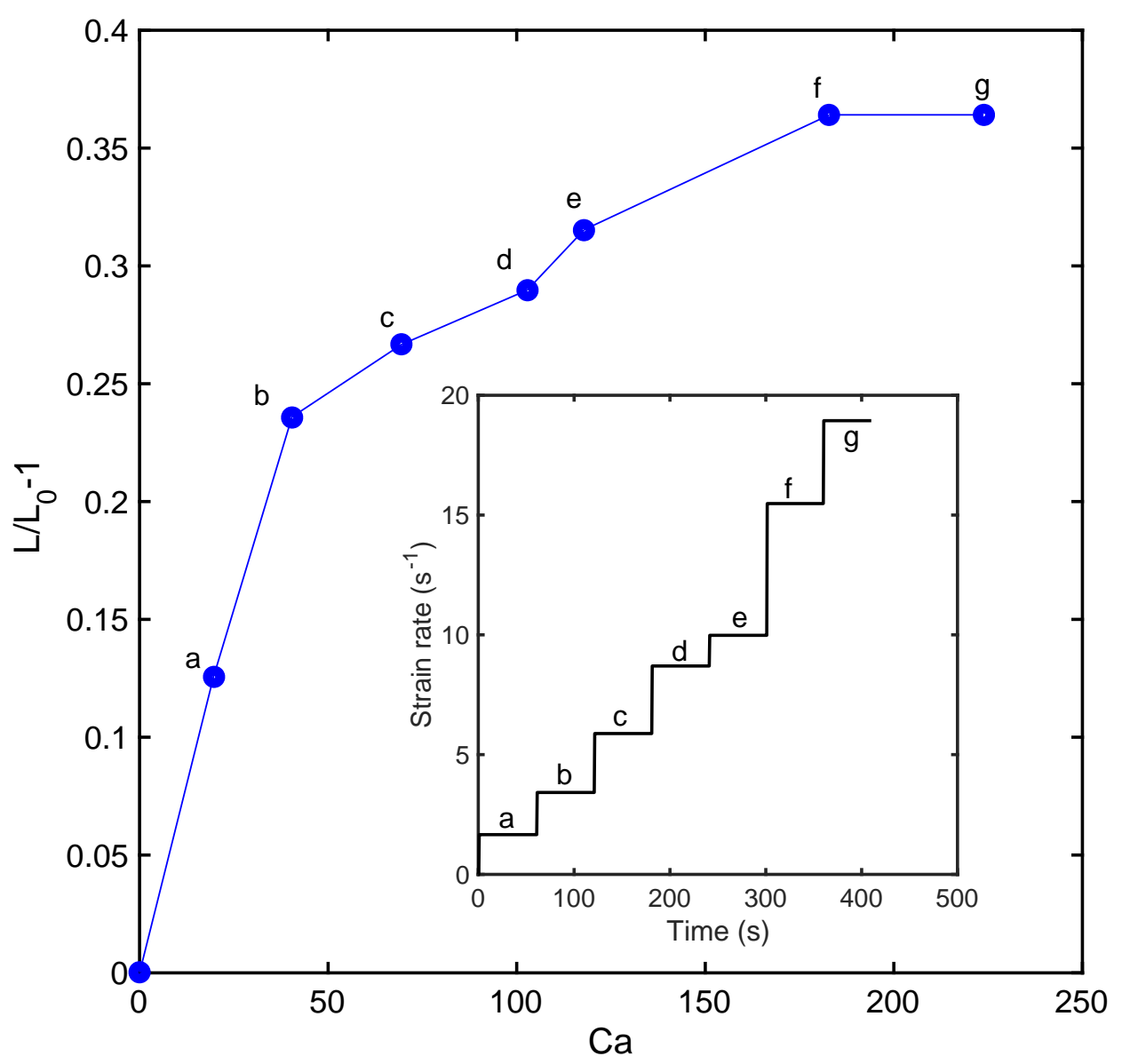

FIG. S2. Steady-state aspect ratio as a function of increasing Capillary number for a vesicle with reduced volume $\nu=0.91$. Inset shows the fluid strain-rate for each level of steady-state aspect ratio deformation. 


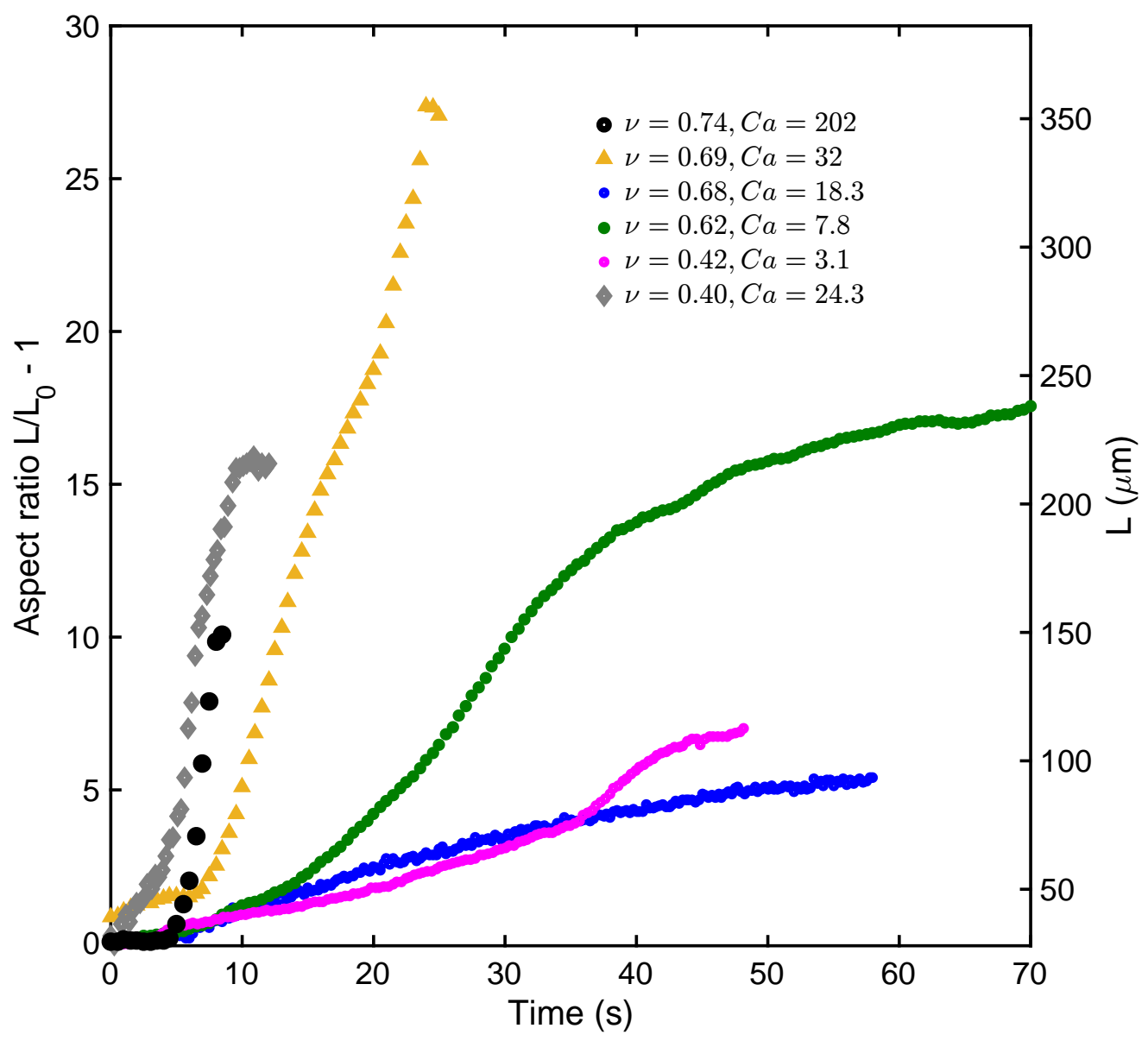

FIG. S3. Characteristic transient stretching trajectories for a wide range of capillary number and reduced volume at $\lambda=0.1$. 


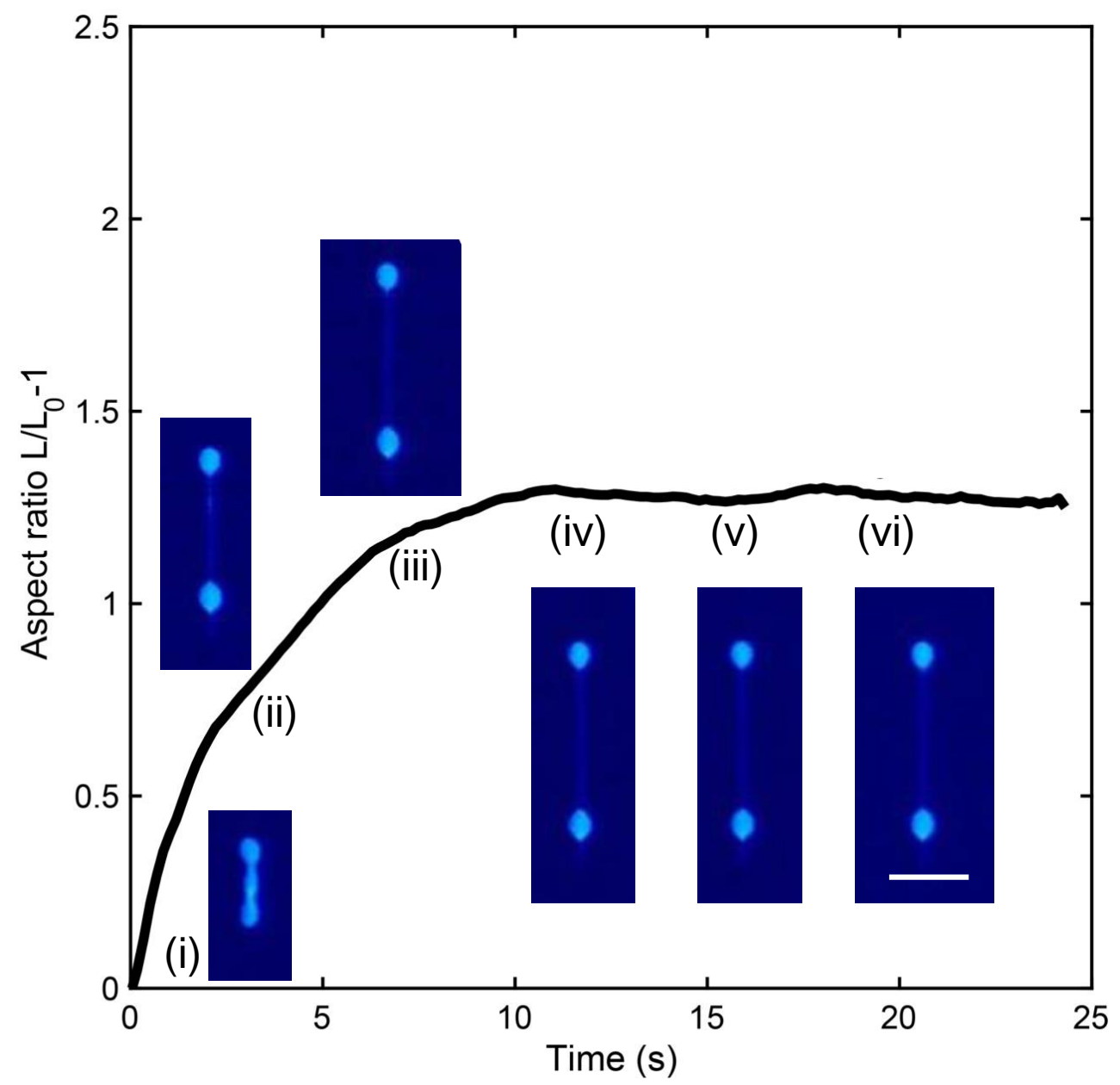

FIG. S4. Transient stretching of a deflated vesicle with reduced volume $0.49 \pm 0.01$ at $C a=2.3$. Aspect ratio $A_{r}$ of the vesicle as a function of time under a step strain rate. Snapshots of the vesicle during stretching at various time instants corresponding to the roman numerals is shown. Scale bar is $25 \mu \mathrm{m}$. 


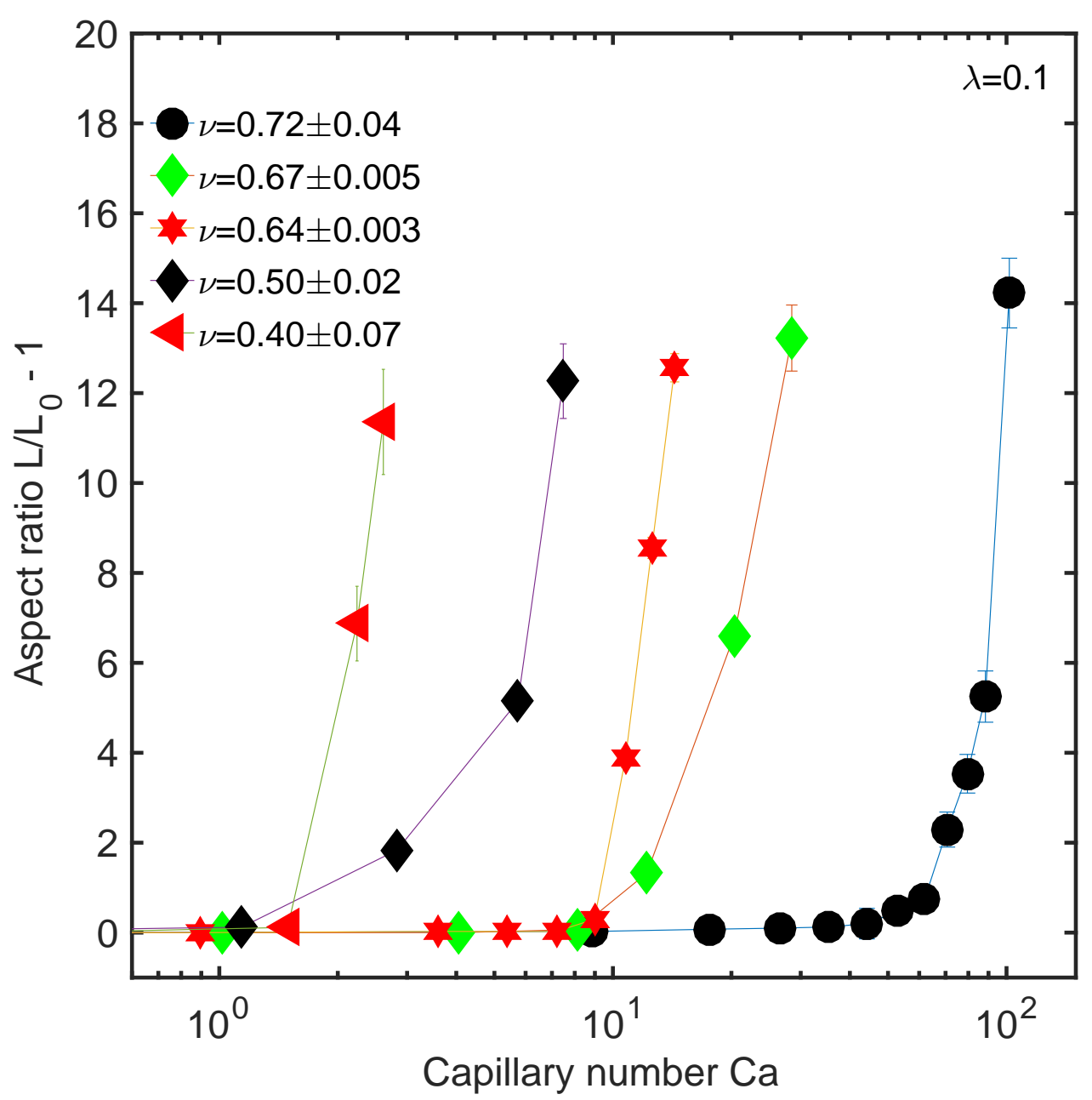

FIG. S5. Steady-state aspect ratio of vesicles as a function of capillary number for several characteristic reduced volumes at a viscosity ratio $\lambda=0.1$. 

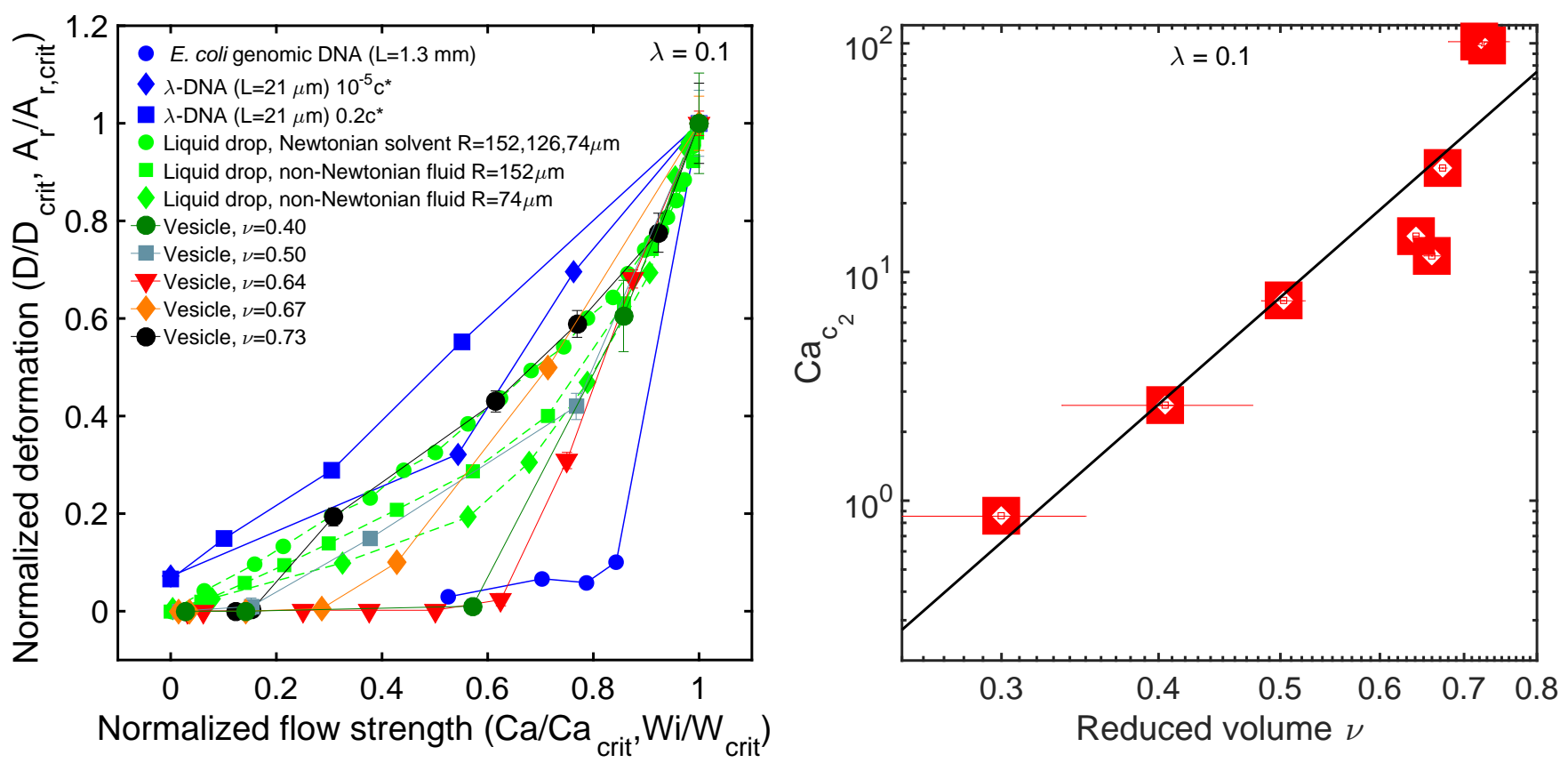

FIG. S6. Characterization of second critical capillary number $C a_{c_{2}}$ for viscosity ratio $\lambda=0.1$. (A) Normalized aspect ratio, fractional extension and deformation parameter for vesicles, polymers and liquid drops as a function of normalized flow strength $\left(\mathrm{Ca} / \mathrm{Ca}_{c_{2}}\right.$ for vesicles, $\mathrm{Ca} / \mathrm{Ca} a_{\text {crit }}$ for drops, $W i / W i_{c r i t}$ for polymers). Error bars represent \pm 1 standard deviation from the mean value. (B) Log-log plot of $C a_{c_{2}}$ as a function of reduced volume $\nu$ for viscosity ratio $\lambda=0.1$. The black line is a fit of the form $C a_{c_{2}}=a \nu^{\beta}$ where $a$ is a constant and $\beta$ is the power-law exponent. We find $\beta=4.9 \pm 0.4$ at $\lambda=0.1$ 


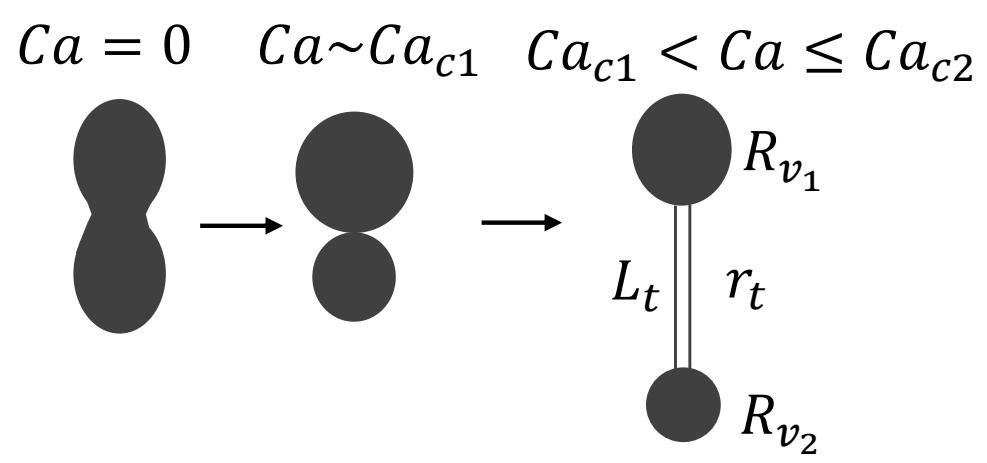

FIG. S7. Schematic of vesicle shapes during stretching by flow at different capillary numbers. 

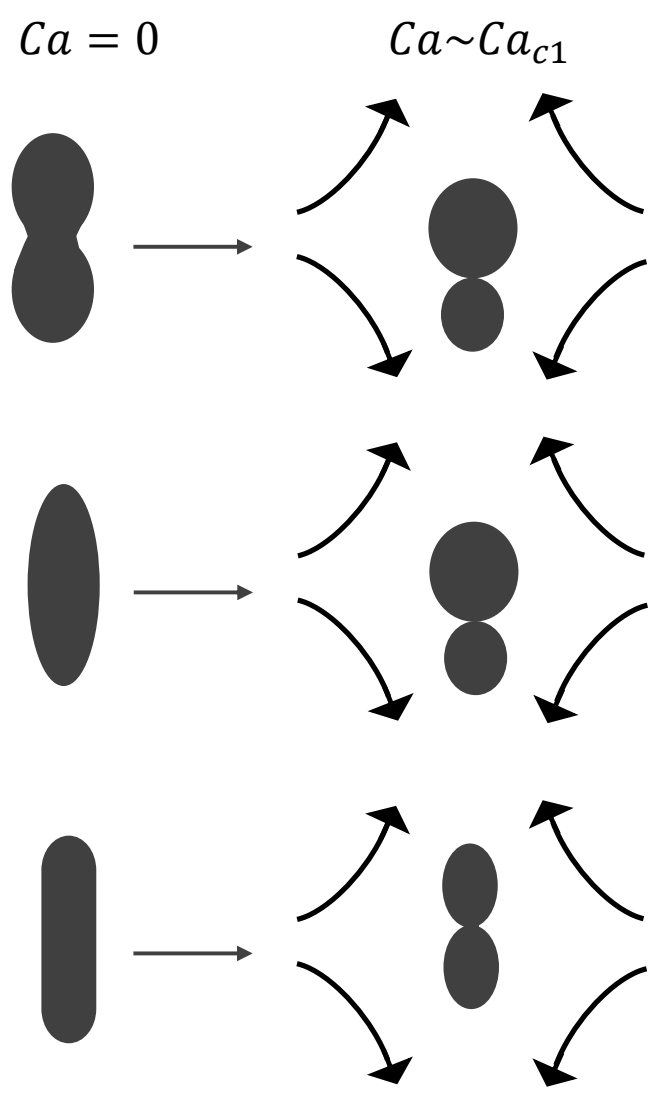

\section{Equilibrium Onset of conformation shape change}

FIG. S8. Pathways of transient vesicle shape, immediately at the onset of conformation change under flow for a biconcave, spheroid and tubular shape. 
* To whom correspondence must be addressed: cms@illinois.edu

[1] R. Dimova, U. Seifert, B. Pouligny, S. Förster, and H.-G. Döbereiner, Hyperviscous diblock copolymer vesicles, The European Physical Journal E 7, 241 (2002).

[2] D. Kumar, C. M. Richter, and C. M. Schroeder, Double-mode relaxation of highly deformed anisotropic vesicles, Physical Review E 102, 010605 (2020).

[3] R. Dimova and C. Marques, The Giant Vesicle Book (CRC Press, 2019).

[4] D. Kumar, C. M. Richter, and C. M. Schroeder, Conformational dynamics and phase behavior of lipid vesicles in a precisely controlled extensional flow, Soft Matter 16, 337 (2020).

[5] V. Kantsler, E. Segre, and V. Steinberg, Critical dynamics of vesicle stretching transition in elongational flow, Phys. Rev. Lett. 101, 048101 (2008).

[6] J. Sanborn, K. Oglkecka, R. S. Kraut, and A. N. Parikh, Transient pearling and vesiculation of membrane tubes under osmotic gradients, Faraday Discuss. 161, 167 (2013).

[7] H. Zhou, B. B. Gabilondo, W. Losert, and W. van de Water, Stretching and relaxation of vesicles, Physical Review E 83, 011905 (2011).

[8] U. Seifert, Fluid membranes in hydrodynamic flow fields: Formalism and an application to fluctuating quasispherical vesicles in shear flow, The European Physical Journal B-Condensed Matter and Complex Systems 8, 405 (1999).

[9] U. Seifert, Configurations of fluid membranes and vesicles, Adv. Phys. 46, 13 (1997). 